\title{
Clinical responses in patients with advanced colorectal cancer to a dendritic cell based vaccine
}

\author{
STEFAN K. BURGDORF ${ }^{1}$, ANDERS FISCHER ${ }^{1}$, PETER S. MYSCHETZKY ${ }^{2}$, SIGNE B. MUNKSGAARD ${ }^{1}$, \\ MAI-BRITT ZOCCA ${ }^{3}$, MOGENS H. CLAESSON ${ }^{4}$ and JACOB ROSENBERG ${ }^{1}$ \\ Departments of ${ }^{1}$ Surgical Gastroenterology and ${ }^{2}$ Radiology, University Hospital of Copenhagen, \\ Gentofte; ${ }^{3}$ DanDrit Biotech, Symbion Science Park, Fruebjergvej 3, Copenhagen; \\ ${ }^{4}$ Faculty of Health Sciences, University of Copenhagen, Denmark
}

Received June 2, 2008; Accepted July 17, 2008

DOI: 10.3892/or_00000145

\begin{abstract}
Patients with disseminated colorectal cancer have a poor prognosis. Preliminary studies have shown encouraging results from vaccines based on dendritic cells. The aim of this phase II study was to evaluate the effect of treating patients with advanced colorectal cancer with a cancer vaccine based on dendritic cells pulsed with an allogenic tumor cell lysate. Twenty patients with advanced colorectal cancer were consecutively enrolled. Dendritic cells (DC) were generated from autologous peripheral blood mononuclear cells and pulsed with allogenic tumor cell lysate containing high levels of cancer-testis antigens. Vaccines were biweekly administered intradermally with a total of 10 vaccines per patient. CT scans were performed and responses were graded according to the RECIST criteria. Quality of life was monitored with the SF-36 questionnaire. Toxicity and adverse events were graded according to the National Cancer Institute's common Toxicity Criteria. Four patients were graded with stable disease. Two remained stable throughout the entire study period. Analysis of changes in the patients' quality of life revealed stability in the subgroups: 'physical function' ( $\mathrm{p}=0.872)$, 'physical role limitation' $(\mathrm{p}=0.965)$, 'bodily pain' ( $\mathrm{p}=0.079)$, 'social function' $(\mathrm{p}=0.649)$, 'emotional role limitation' ( $\mathrm{p}=0.252)$ and 'mental health' $(\mathrm{p}=0.626)$. The median survival from inclusion was 5.3 months (range 0.2-29.2 months) with one patient still being alive almost 30 months after inclusion in the trial. Treatment with this DC-based cancer vaccine was safe and non-toxic. Stable disease was found in $24 \%(4 / 17)$ of the patients. The quality of life remained for most categories high and stable throughout the study period.
\end{abstract}

Correspondence to: Dr Stefan K. Burgdorf, Department of Surgical Gastroenterology, Gentofte University Hospital, Niels Andersens Vej 65, DK-2900 Hellerup, Denmark

E-mail: stefan@stefanburgdorf.dk

Key words: dendritic cells, cancer vaccines, colorectal cancer, quality of life

\section{Introduction}

Cancer is the second leading cause of death and colorectal cancer (CRC) is the third leading cause of death of cancer in both men and women in the USA (1). During recent years there have been major advances in the treatment of CRC. The surgical resections, radiation therapies, chemo- and antibody-based therapies are more effective than ever, but the prognosis for advanced CRC is still poor. The overall survival rate is $<10 \%$ (2). Many of the oncological treatments are lifeprolonging, but are in many cases complicated by poor quality of life and unacceptable adverse effects (2-7). Development of new and even more effective and less toxic treatments are therefore of great importance. Immunotherapy has been developed and tested for years $(8,9)$, with one of the newer encouraging treatment options being dendritic cell (DC)based vaccines (10-12).

The approaches of cancer vaccines are very different from traditional oncological treatments. Where traditional chemotherapy, as a negative side-effect, depresses the patients' immune system, the cancer vaccines attempt to strengthen the patients' immune system in a focused attack on the cancer cells. Cancer vaccines are not toxic and therefore are more tolerable than traditional oncological treatments. The efficacy of the DC-based cancer vaccines has been studied in various types of cancers and has in some cases been very encouraging (10-13). The most promising clinical responses have been described in patients with malignant melanoma and non-Hodgkin's lymphoma (10).

The aim of the present study was to evaluate the effect of treating patients with advanced colorectal cancer with a cancer vaccine based on dendritic cells pulsed with an allogenic tumor cell lysate.

\section{Patients and methods}

Patients. Twenty patients with disseminated colorectal cancer were consecutively enrolled in this phase II study. The inclusion criteria were biopsy verified colonic or rectal cancer with distant metastases, age $\geq 25$ and $\leq 75$ years at inclusion, no radio- or chemotherapy within 6 weeks prior to inclusion, performance status $\geq 2$ (WHO performance status scale), expected survival at inclusion $>4$ months, blood 
samples showing adequate hepatic, renal, haematopoietic and coagulatory function at the time of inclusion, normal EKG and preserved pulmonary function. The exclusion criteria were immune suppressing treatment (e.g., systemic corticosteroid) up to 2 months before inclusion, uncontrolled serious infections, participation in other clinical trials during the last 6 weeks and, for women, pregnancy or lactation.

Study design. Each of the included patients was treated according to a standard vaccination schedule with a total of ten vaccines administered biweekly. The vaccines were injected intradermally on the proximal thigh. Every vaccine consisted of four injections, two on the right and two on the left thigh. A total of $3-5 \times 10^{6}$ DCs were injected. The DCs used for the vaccines were cultured in vitro from $200 \mathrm{ml}$ freshly drawn peripheral blood and pulsed with an allogenic tumor cell lysate. Preparation and pulsing of DCs and preparation of the cell lysate, derived from a tumor antigen (MAGE) expressing melanoma cell line, have previously been described (14). The study design included four CT scans with one at inclusion, one after five given vaccines, one after all ten vaccines and finally a CT scan 6 months after the tenth vaccine. Responses were assessed by a senior radiologist using the Response Evaluation Criteria in Solid Tumors (RECIST) (15). DTH was performed before intervention and again after 5 vaccinations. The specific antigen for the DTH test was the allogenic tumor cell lysate. We used Tuberculin as positive control and $\mathrm{NaCl}$ as negative control. Results were measured after 3 days and positive result was defined as an induration of $\geq 6 \mathrm{~mm}$. Before every vaccination the patients independently filled out the quality of life (SF-36) questionnaire $(16,17)$ and had blood samples taken. Uses of other medications were monitored throughout the entire study period. Adverse events were monitored and graded according to the National Cancer Institute's Common Toxicity Criteria (NCI's CTC). After inclusion all patients had two ultrasonic assisted needle biopsies $(0.9 \mathrm{~mm})$ made from a distant metastasis (mostly hepatic metastases) and the biopsies underwent an RT-PCR analysis for expression of tumor associated MAGE antigens. We have previously described this procedure in detail (14).

The study was performed at the Department of Surgical Gastroenterology at Gentofte University Hospital, Copenhagen, Denmark according to ICH Guidelines for Good Clinical Practice (European Directive on GCP 2001/20/EC). The study was registered at ClinicalTrials.gov (identification number: NCT00311272). All patients gave at the inclusion their signed informed consent according to Danish law and Good Clinical Practice (GCP). The study was approved by the local ethics committee, the Danish Health Authorities, the GCP unit at Copenhagen University Hospital, and by the Danish Data Protection Agency.

Quality of life. The SF-36 ('short form' with 36 questions) was used in this study $(16,17)$. After initial instruction the patients independently filled out the questionnaire, i.e., before they talked to the investigating doctor. The questionnaire covers the following eight categories: physical function, physical role limitation, bodily pain, general health perceptions, vitality, social function, emotional role limitation, mental health. Thus, the SF-36 covers both physical and mental
Table I. Patient characteristics at baseline $(n=20)$.

\begin{tabular}{lcc}
\hline Characteristics & No. of patients & $\%$ \\
\hline Sex & & \\
Female & 10 & 50 \\
Male & 10 & 50
\end{tabular}

Median age (range)

$60(40-72)$ years

WHO performance

status at inclusion

$\begin{array}{rrr}0 & 10 & 50 \\ 1 & 9 & 45 \\ 2 & 1 & 5\end{array}$

Expression of MAGE

antigens $(n=19)$

$\begin{array}{rrr}0 & 4 & 21 \\ 1 & 1 & 5 \\ 2 & 0 & 0 \\ 3 & 0 & 0 \\ 4 & 0 & 5 \\ 5 & 1 & 21 \\ 6 & 4 & 47\end{array}$

Median (range)

$5(0-6)$

Median time from initial

diagnosis to inclusion (range) 36.4 (11.6-73.0) months

Initial CEA level (range)

$(\mathrm{n}=18)$

$84.1(3.9-3405.0) \mu \mathrm{g} / 1$

quality of life. All of the answers were, according to the manual, scored and transformed to a scale from 0 to 100 , with higher scores indicating better status.

Statistical analysis. The changes in the patients' quality of life throughout the study period were analysed using Friedman's test. Statistical significance was determined at the $\mathrm{p}<0.05$ level. The overall survival time was estimated using the KaplanMeier test. Comparison of overall survival for patients with stable disease versus patients with progressive disease was tested with Breslows (Generalized Wilcoxon) test of equality. Changes in Serum-CEA levels were monitored and analysed using Wilcoxon signed ranks test. To compensate for multiple statistical analyses, Bonferroni correction was used when necessary.

\section{Results}

From November 2004 to April 2006 we screened 36 patients of whom we included 20 in the study. The patients who were not included were primarily rejected because of a low performance status (WHO performance status score $>2$ ) or 


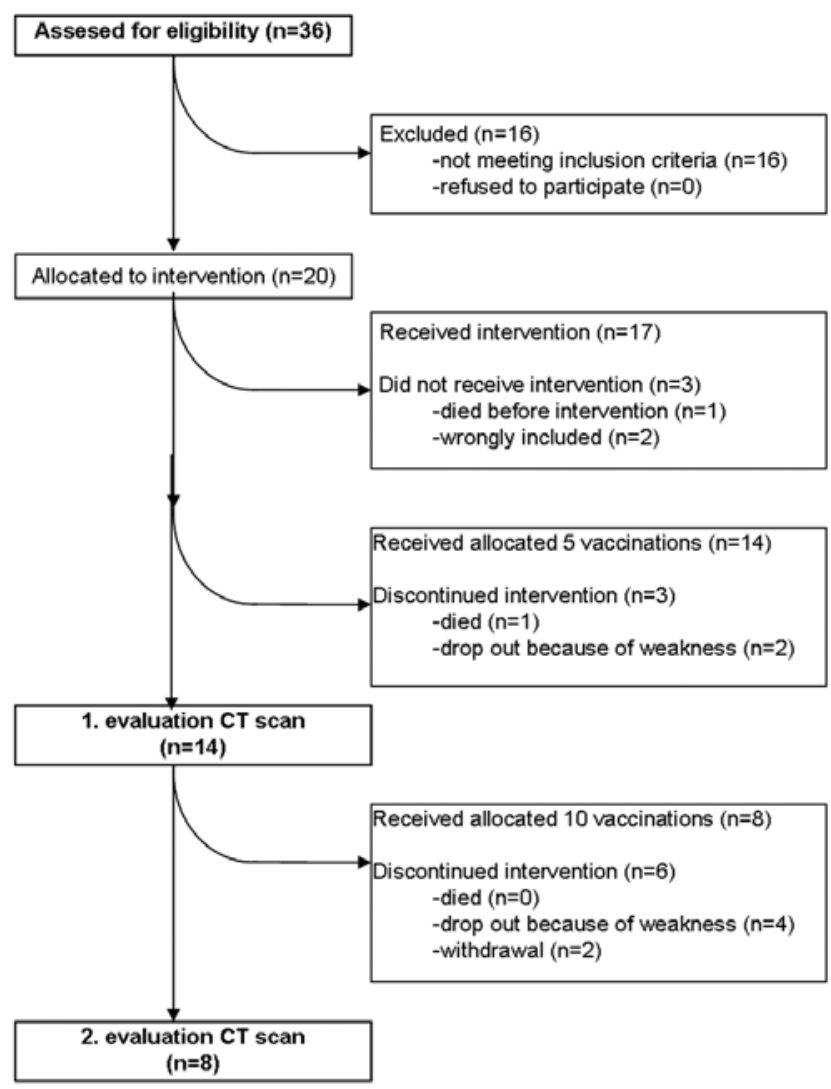

Figure 1. Study profile.

death before the day of screening. Three patients were not included in the efficacy analysis of tumor response because they dropped out of the study before the second CT scan. Two of these patients were wrongly included and the third died before receiving intervention.

Patient characteristics. Patient characteristics are summarised in Table I. All of the included patients had prior to inclusion received various treatments for their cancer disease (some of them up to four different chemotherapeutic regimes, radiotherapy and surgery) and there were no indication for further oncological or surgical treatment. The expressions of tumor associated antigens (MAGE A-1, A-3, A-4, A-6, A-10 and A-12) in needle biopsies from the patients' tumors were determined at inclusion. The majority of the patients $(79 \%)$ expressed one or more of the MAGE antigens, and $47 \%$ of the patients expressed all 6 MAGE antigens (Table I).

Vaccines and CT scans. The total number of vaccines administered was 133. Eight patients received all of the ten scheduled vaccines. One patient received further treatment with additional monthly vaccination because the patient was evaluated to have stable disease after the scheduled ten vaccines. Three of the patients did not receive any vaccines - two of them were wrongly included (one turned out not to have primary cancer focus in colon or rectum, but in the lungs and one was excluded because of corticosteroid use) and one died before the first vaccine. Six patients had early drop out of the study and did not go through the second CT scan and are therefore not evaluable with the RECIST criteria. Eight patients made it to the third CT scan and one patient had all four CT scans done (Fig. 1).

Clinical responses. The best observed response, according to the RECIST criteria, was stable disease. At the first evaluating CT scan four patients were categorised with stable disease and at the second evaluating CT scan two of these patients still had stable disease and one of them received additional monthly vaccines because of the remaining stability in the disease. Table II shows results from RECIST analysis of the CT scans of these four patients. The baseline CT scan and the first evaluating CT scan from one of the stable patients are shown in Fig. 2. The measured tumor (target lesion) was located in the left lung. On the same slide, close to the measurable target lesion, there was a non-measurable nontarget lesion that had disappeared at the next CT scan.

Survival was defined as time from inclusion or time of initial diagnosis to death. The overall survival was estimated and visualised on a Kaplan-Meier curve (Fig. 3a and b). The median survival from inclusion was 5.3 months (range 0.2-29.2 months, 95\% CI 4.3-6.3 months) (Fig. 3a). The median survival from initial diagnosis was 43.1 months (range 11.6-73.0 months, 95\% CI 29.9-56.3 months) (Fig. 3b). At the initial diagnosis 10 patients $(53 \%)$ had disseminated

Table II. Results from RECIST analysis of the four patients with stable disease. ${ }^{\mathrm{a}}$

\begin{tabular}{|c|c|c|c|c|c|c|c|}
\hline \multirow[b]{2}{*}{ Patient } & \multirow{2}{*}{$\begin{array}{c}\text { Baseline CT } \\
\frac{\text { scan }}{\text { Sum of longest }} \\
\text { diameters }(\mathrm{cm})\end{array}$} & \multicolumn{3}{|c|}{ First evaluable CT scan } & \multicolumn{3}{|c|}{ Second evaluable CT scan } \\
\hline & & $\begin{array}{l}\text { Sum of longest } \\
\text { diameters }(\mathrm{cm})\end{array}$ & $\begin{array}{l}\text { New lesions } \\
(\mathrm{cm})\end{array}$ & Status & $\begin{array}{c}\text { Sum of longest diameters } \\
\text { diameters }(\mathrm{cm})\end{array}$ & New lesions & Status \\
\hline 6 & 2.41 & 2.55 & No & SD & 3.18 & Yes & PD \\
\hline 8 & 19.48 & 19.64 & No & SD & 21.87 & No & SD \\
\hline 10 & 6.19 & 6.72 & No & SD & 8.42 & Yes & PD \\
\hline 19 & 1.07 & 0.95 & No & SD & 0.95 & No & SD \\
\hline
\end{tabular}

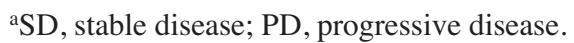




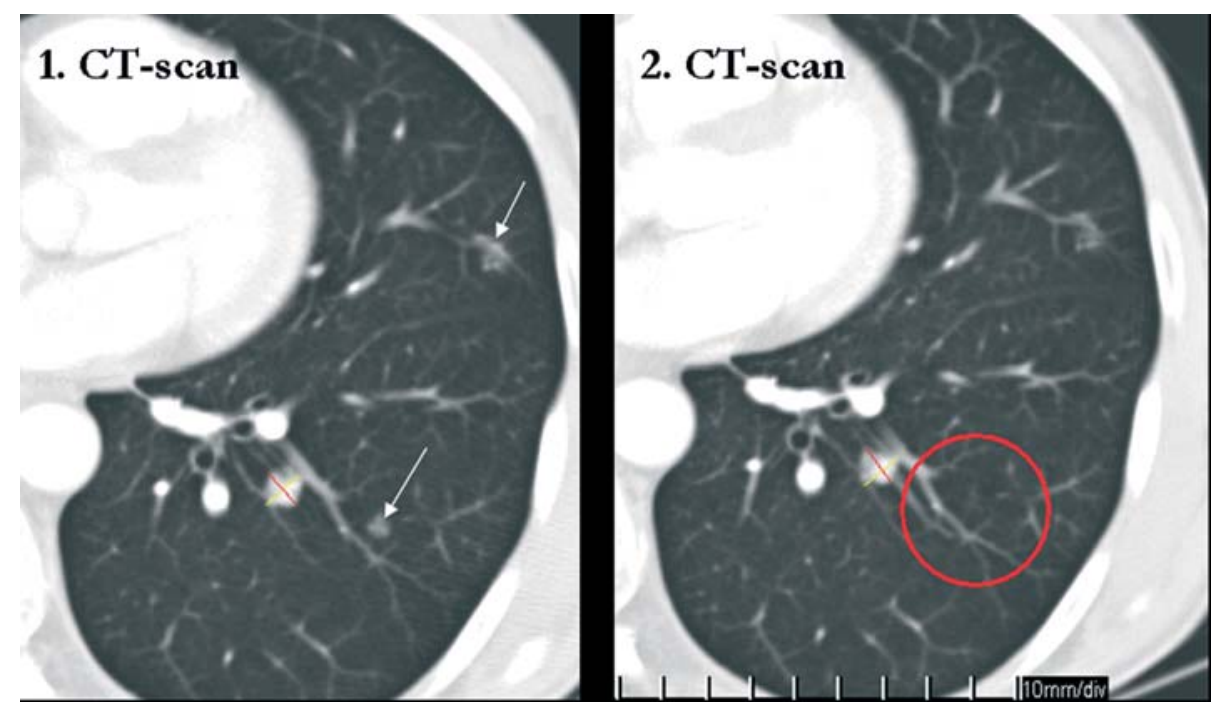

Figure 2. Baseline and first evaluating CT scan of a patient with stable disease. Left, baseline CT scan showing a target lesion measured with a longest diameter of $1.07 \mathrm{~cm}$ and two non-target lesions (arrows). Right, first evaluation CT scan showing the same target lesion measured with a longest diameter of $0.95 \mathrm{~cm}$ and disappearance of one of the non-target lesions (circle).

$\mathbf{a}$

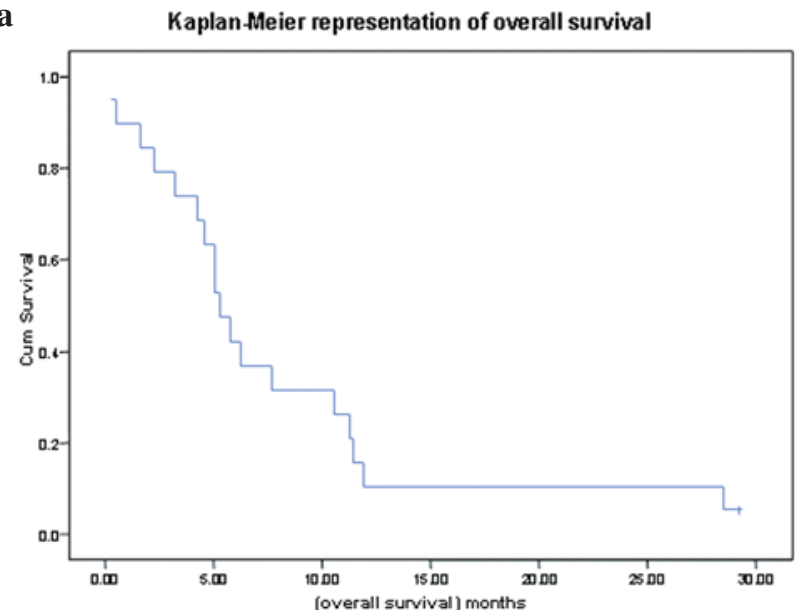

c

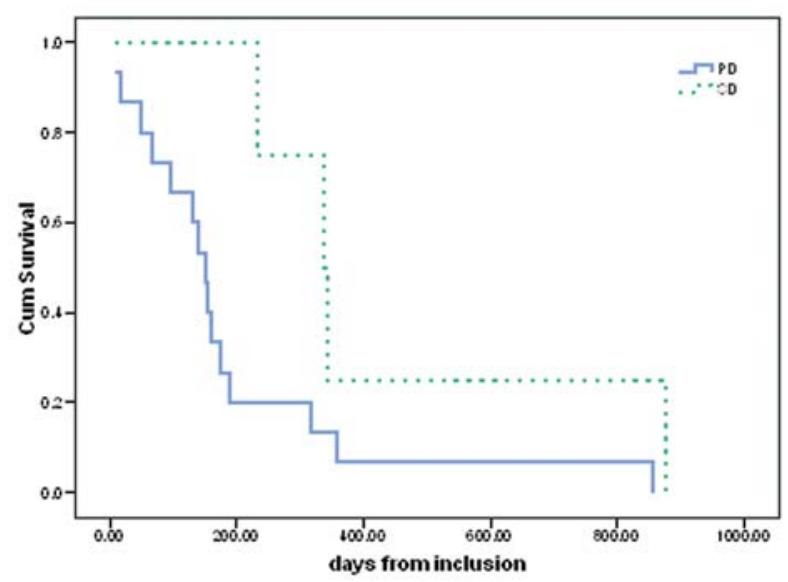

disease. At time of submission of this report one patient was still alive.

Comparison of overall survival for patients with stable disease versus patients with progressive disease showed b

Survival Function

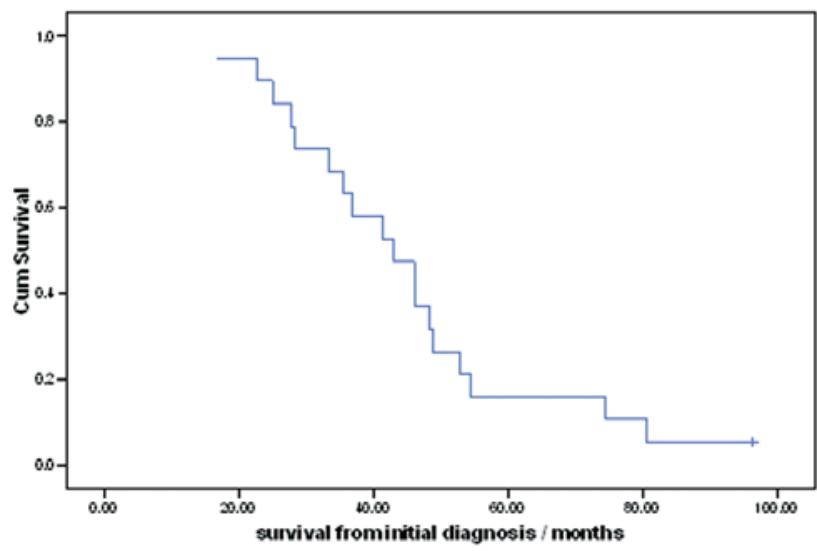

Figure 3. a, Kaplan-Meier curve of median survival from inclusion. b, KaplanMeier curve of median survival from initial diagnosis, where $53 \%$ of the patients had disseminated disease. c, Kaplan-Meier curve of median survival from inclusion for patients with progressive disease (PD) and patients with stable disease (SD).

that patients with stable disease lived significantly longer $(\mathrm{p}=0.038)$ than patients with progressive disease (Fig. 3c).

$D T H$. None of the patients showed positive DTH reactions neither before nor during treatment. Seven of the patients did not show positive reaction to the positive tuberculin control either. This may be due to a general low or suppressed immunity among the patients.

Quality of life. Changes in the patients' self-reported quality of life during the study period, assessed by the SF-36 questionnaire, were estimated using Freidman's statistical analysis. Graphical presentation of the results from the analysis of quality of life is shown in Fig. 4. There were no significant changes in the patients' 'physical function' $(\mathrm{p}=0.872)$, 'physical role limitation' $(\mathrm{p}=0.965)$, 'bodily pain' $(\mathrm{p}=0.079)$, 'social 

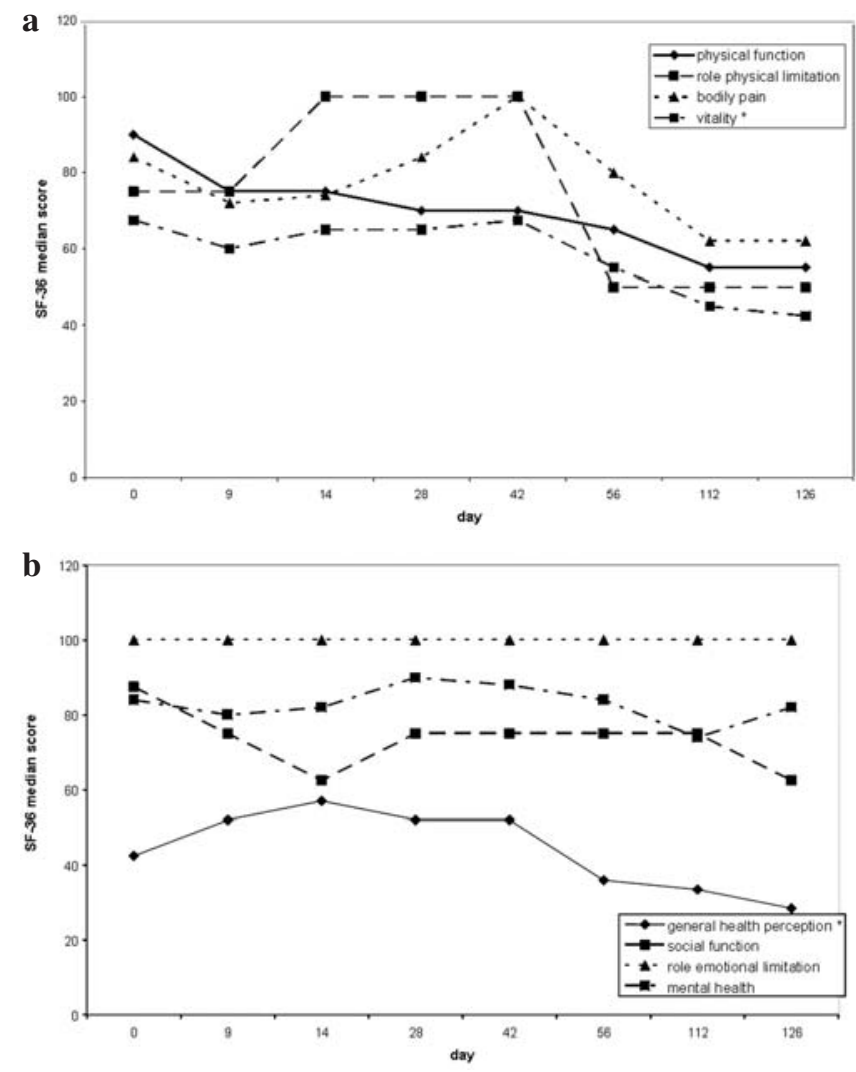

Figure 4. Changes in quality of life during the study period estimated with Friedman's statistical analysis. The median values are shown in the plots. There were no significant changes in the patients' 'physical function' ( $\mathrm{p}=0.872$, 'physical role limitation' ( $\mathrm{p}=0.965)$, 'bodily pain' $(\mathrm{p}=0.079)(\mathrm{a})$, 'social function' ( $p=0.649)$, 'emotional role limitation' $(p=0.252)$ and 'mental health' $(p=0.626)(b)$. There were a significant lower score at the end of the study concerning 'general health perception' ( $\mathrm{p}=0.006)$ (b) and 'vitality' $(\mathrm{p}=0.011)(\mathrm{a})$

function' $(p=0.649)$, ‘emotional role limitation' $(p=0.252)$ and 'mental health' $(\mathrm{p}=0.626)$. There was a significantly lower score towards the end of the study concerning 'general health perception' $(p=0.006)$ and 'vitality' $(p=0.011)$.
CEA levels. Serum CEA levels were measured before, in the middle of vaccination regime (i.e., after 5 vaccines), and at the end of the treatment (i.e., after 10 vaccines). The median CEA levels are shown in Fig. 5. The figure shows the CEA levels for all of the patients pooled in one group and for two subgroups with patients with progressive disease in one group and patients with stable disease in the other group. Tested with Wilcoxon signed ranks test there was for the entire cohort a significantly higher $(\mathrm{p}=0.004)$ serum CEA level after 5 vaccines compared to before intervention and a significantly lower $(\mathrm{p}=0.007)$ serum CEA level after the entire vaccine regime (10 vaccines) than before intervention. For the subgroup with progressive disease the median CEA level at inclusion was $183 \mu \mathrm{g} / \mathrm{l}$ (range 4.3-3405 $\mu \mathrm{g} / \mathrm{l}$ ) and there was a significantly higher $(\mathrm{p}=0.028)$ serum CEA level after the entire vaccine regime than before intervention. For the subgroup with stable disease the median CEA level at inclusion was $12.1 \mu \mathrm{g} / \mathrm{l}$ (range 3.9-22.6 $\mu \mathrm{g} / \mathrm{l}$ ) and there was no significant change $(\mathrm{p}=0.144)$ in the serum CEA level when comparing prevaccine levels with post intervention levels.

Adverse effects and toxicity. We have in our phase I report (14) shown that treatment with this DC-based cancer vaccine was safe and non-toxic. The ongoing registration of adverse events following this former report has confirmed this conclusion, since we have not observed any serious adverse events or toxicity related to the treatment with the DC-based vaccine.

\section{Discussion}

In this phase II study we have estimated the effects of treating patients with disseminated CRC, who had no indication for further radio-, chemo- or surgical therapy, with a newly developed DC-based vaccine. The clinical response rates according to the RECIST criteria were limited. We did not find any partial- or complete responses, however of a total of 20 included and 17 evaluable patients we found four patients with stable disease of whom two remained stable throughout

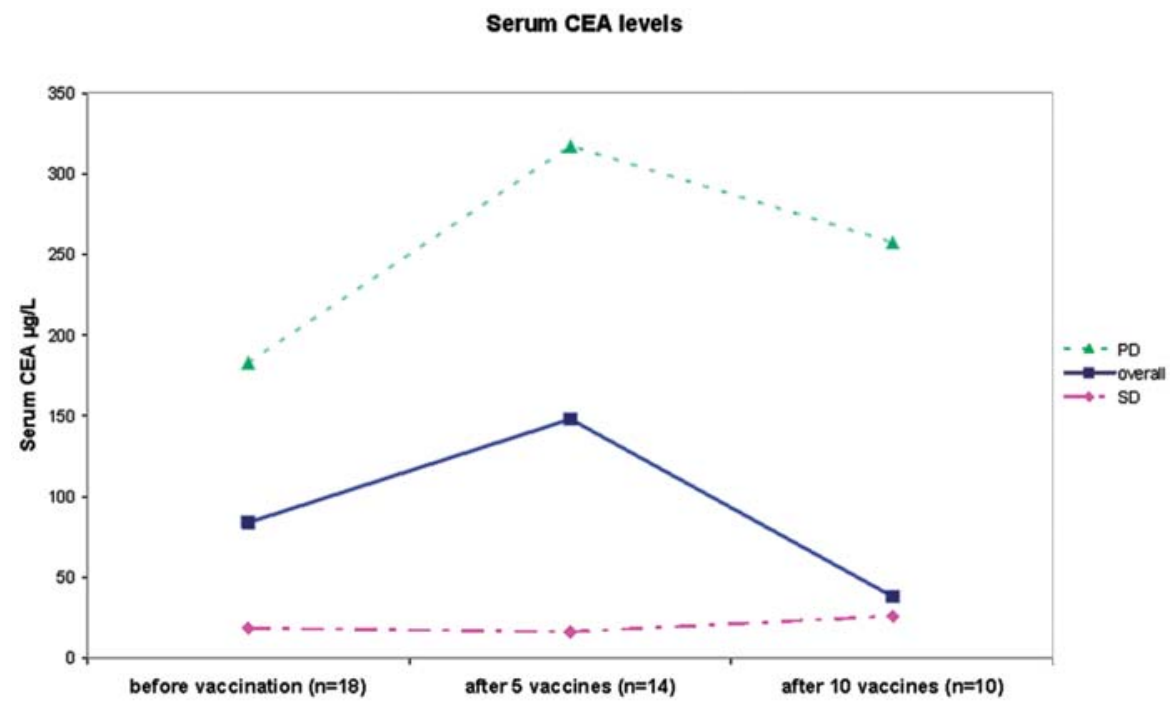

Figure 5. The diagram shows the median serum CEA levels for the entire cohort (overall), for the subgroup of progressive patients (PD) and for the subgroup of stable patients $(\mathrm{SD})$ before vaccination, after 5 vaccines and finally after 10 vaccines. 
the entire study period. More than that, we did not observe any adverse effects or toxic reactions related to the treatment and the patients' quality of life remained for most categories of the SF-36 high and stable.

The clinical response rates to DC-based cancer vaccines have in the literature been reported highest for patients with malignant melanoma and non-Hodgkin's lymphoma (10) but encouraging results have also been revealed in some CRC trials (11). At the same time it is stressed, that in studies with disseminated CRC, with patients having a fairly short life expectancy, it is necessary at the same time to look at other end-points than partial and complete responses $(8,11)$. These 'softer' end-points are stable disease, quality of life, toxicity and adverse events profile - all of which are of major importance for the patient with a lethal disease such as disseminated CRC. Furthermore, these small benefits could add up to a clinically essential end-point.

Data from the CEA analysis suggest that there is a correlation between low CEA levels and stable disease. CEA is often used to monitor stability versus activity during conventional CRC treatment and therefore it is not surprising that the CEA levels are lower for the stable disease patients. Interestingly, our data may suggest, that patients with low CEA are the ones who might respond to the vaccine.

Expression of MAGE antigens in our study cohort was similar to that reported previously (18). Our hypothesis from the beginning was that the patients with high expression of MAGE antigens (the same as in the vaccine) would be most likely to respond to the treatment. Interestingly, we have noted that the tumor of one of the patients with stable disease was MAGE-negative. If stable disease in this patient was induced by the vaccine, this might suggest that multiple epitopes exist and are active factors in our allogenic tumor lysate used for vaccine production (14). Recent PCR-driven data from the actual melanoma cell line, from which the vaccine lysate is derived, has confirmed the presence of a number of other tumor associated antigens (DanDrit Biotech, unpublished data).

In many cancer trials including those with disseminated CRC trials, the frequency of reported grade 3 and 4 toxicity (NCI's CTC) directly related to the therapeutic agent is considerable $(2-7,19,20)$. The treatment with DC-based vaccines is, compared with many other cancer treatments, associated with remarkably few adverse effects if any, and consequently the physical and mental strains on the patients are noteworthy lower.

During the past, more studies have been carried out using dendritic cell vaccination in CRC. A recent meta-analysis by Nagorson and Thiel (11) with a total of 527 patients with advanced CRC in 32 studies estimated the clinical and immunological responses to active specific cancer vaccines. For the entire population of 527 patients, one complete response and four partial responses were reported adding up to an objective response rate of $0.9 \%$. Four of these five clinical responses were all reported in the same study (21). The authors of the mentioned meta-analysis suggested a new term called 'clinical benefit rate' defined as the sum of complete responses, partial responses, mixed responses and stable disease rates. This clinical benefit rate was for all patients estimated to be $11.2 \%$ (11). For DC-based vaccines alone the clinical benefit rate was $17 \%(\mathrm{n}=70)$. In our study, this clinical benefit rate would comparably be $24 \%$ (4/17).

Treating patients with disseminated CRC is a complicated affair. The disease is, like most other advanced cancers, unmerciful and the prognosis is very poor. Many patients suffer with devastating adverse effects from the treatment they receive and their life expectancy, in spite of treatment, is short. Cancer vaccines may change some of these pessimistic aspects for some patients. The toxicity and adverse events related to the vaccines are for sure less incriminating than traditional oncological treatment. If treatment with cancer vaccines has clinical effects, which is documented in some cases, it is worth working on the implementation of vaccination in the clinical practice. This is especially supported by our findings of high and stable quality of life in most patients during treatment.

The low level of clinical efficacy in the present and in similar vaccine studies might reflect the severely hampered immune functions in patients with large disseminated tumor burdens (22-24) and the many potential tumor immune evasion mechanisms (25) which negatively affect an effective vaccine-induced $\mathrm{T}$ cell-mediated immune response against the tumors in these patients. To be more effective, future tumor vaccine treatments should include patients with less tumor burdens and attempts to modify immune homeostasis of the patients such as e.g., antibody or chemotherapy induced deletion of counteracting $\mathrm{T}$ regulatory cells (25) prior to vaccination.

\section{Acknowledgements}

This work was supported by a grant from The Aase and Ejnar Danielsens Foundation.

\section{References}

1. Jemal A, Murray T, Ward E, et al: Cancer statistics, 2005. CA Cancer J Clin 55: 10-30, 2005.

2. Goldberg RM: Advances in the treatment of metastatic colorectal cancer. Oncologist 10 (Suppl. 3): 40-48, 2005.

3. Zampino MG, Magni E, Massacesi C, et al: First clinical experience of orally active epidermal growth factor receptor inhibitor combined with simplified FOLFOX6 as first-line treatment for metastatic colorectal cancer. Cancer 110: 752-758, 2007.

4. Ju JH, Chang SC, Wang HS, et al: Changes in disease pattern and treatment outcome of colorectal cancer: a review of 5,474 cases in 20 years. Int J Colorectal Dis 22: 855-862, 2007.

5. Arndt V, Merx H, Stegmaier C, Ziegler H and Brenner H: Restrictions in quality of life in colorectal cancer patients over three years after diagnosis: a population based study. Eur J Cancer 42: 1848-1857, 2006.

6. McWilliams RR, Goetz MP, Morlan BW, et al: Phase II trial of oxaliplatin/irinotecan/5-fluorouracil/leucovorin for metastatic colorectal cancer. Clin Colorectal Cancer 6: 516-521, 2007.

7. Nakata B, Sowa M, Tsuji A, et al: Continuous infusion of 5fluorouracil with versus without low-dose, consecutive administration of cisplatin in advanced colorectal cancer. A prospective randomized phase II study. J Exp Clin Cancer Res 26: 51-60, 2007.

8. Rosenberg SA, Yang JC and Restifo NP: Cancer immunotherapy: moving beyond current vaccines. Nat Med 10: 909-915, 2004.

9. Huber $\mathrm{CH}$ and Wolfel T: Immunotherapy of cancer: from vision to standard clinical practice. J Cancer Res Clin Oncol 130: 367-374, 2004.

10. Cranmer LD, Trevor KT and Hersh EM: Clinical applications of dendritic cell vaccination in the treatment of cancer. Cancer Immunol Immunother 53: 275-306, 2004. 
11. Nagorsen D and Thiel E: Clinical and immunologic responses to active specific cancer vaccines in human colorectal cancer. Clin Cancer Res 12: 3064-3069, 2006.

12. Ridgway D: The first 1000 dendritic cell vaccinees. Cancer Invest 21: 873-886, 2003.

13. Rosenberg SA: Progress in the development of immunotherapy for the treatment of patients with cancer. J Intern Med 250: 462-475, 2001.

14. Burgdorf SK, Fischer A, Claesson MH, et al: Vaccination with melanoma lysate-pulsed dendritic cells, of patients with advanced colorectal carcinoma: report from a phase I study. J Exp Clin Cancer Res 25: 201-206, 2006.

15. Therasse P, Arbuck SG, Eisenhauer EA, et al: New guidelines to evaluate the response to treatment in solid tumors. European Organization for Research and Treatment of Cancer, National Cancer Institute of the United States, National Cancer Institute of Canada. J Natl Cancer Inst 92: 205-216, 2000.

16. Bjorner JB, Thunedborg K, Kristensen TS, Modvig J and Bech P: The Danish SF-36 Health Survey: translation and preliminary validity studies. J Clin Epidemiol 51: 991-999, 1998.

17. Ware JE Jr and Sherbourne CD: The MOS 36-item short-form health survey (SF-36). I. Conceptual framework and item selection. Med Care 30: 473-483, 1992.
18. Gerhardt A, Usener D, Keese M, et al: Tissue expression and sero-reactivity of tumor-specific antigens in colorectal cancer. Cancer Lett 208: 197-206, 2004.

19. De GA, Figer A, Seymour M, et al: Leucovorin and fluorouracil with or without oxaliplatin as first-line treatment in advanced colorectal cancer. J Clin Oncol 18: 2938-2947, 2000.

20. Saltz LB, Cox JV, Blanke C, et al: Irinotecan plus fluorouracil and leucovorin for metastatic colorectal cancer. Irinotecan Study Group. N Engl J Med 343: 905-914, 2000.

21. Liang W, Wang H, Sun TM, et al: Application of autologous tumor cell vaccine and NDV vaccine in treatment of tumors of digestive tract. World J Gastroenterol 9: 495-498, 2003.

22. Klein G and Klein E: Surveillance against tumors - is it mainly immunological? Immunol Lett 100: 29-33, 2005.

23. Yu P, Lee Y, Wang Y, et al: Targeting the primary tumor to generate CTL for the effective eradication of spontaneous metastases. J Immunol 179: 1960-1968, 2007.

24. Reiman JM, Kmieciak M, Manjili MH and Knutson KL: Tumor immunoediting and immunosculpting pathways to cancer progression. Semin Cancer Biol 17: 275-287, 2007.

25. Leen AM, Rooney CM and Foster AE: Improving T cell therapy for cancer. Annu Rev Immunol 25: 243-265, 2007. 